\title{
Determinación de la Aptitud para la Obtención de Aceite de Oliva de Seis Variedades de Aceituna Negra (Olea europaea L.) del Banco de Germoplasma del INPREX UNJBG Tacna
}

\author{
Determination of Aptitude to Obtain Olive Oil from Six \\ Varieties of Black Olive (Olea europaea L.) in the Germplasm \\ Bank from INPREX UNJBG Tacna
}

${ }^{1}$ Enrique Alfonso De Florio Ramirez ${ }^{2}$ Liliana del Carmen Lanchipa Bergamini

\begin{abstract}
RESUMEN:
En el presente trabajo se determinó la aptitud para la obtención de aceite de oliva de seis variedades de aceinuna (Olea europaea L.) del hanco de germoplasma del INPREX - UNJBG. A estas variedades se analizaron caracteristicas del fruto: Indice de madurez, fue óptimo de 3-3.7 (antes del envero): Peso fresco se encontró entre bajo y medio y la relación Pulpa-hueso, entre medio y baja. Respecto al Porcentaje de aceite fue de Empeltre (24.47\%), Arbequina $(15.67 \%)$. Sevillana $(24.67 \%)$, Cornezuelo $(9.12 \%)$. Villalonga $(10.85 \%)$. Azupa $(15.50 \%)$. Al aceite de oliva extra virgen ohtenido de las seis variedades en estudio se realizó análisis fisico químicos: Índice de acidez, indice de Peróxidos, Kra encontrándose lodas dentro de la norma. Por la cantidad y la calidad fisico quimica de aceite producido se ha considerado en las siguientes variedades, la aptitud aceitera en orden decreciente: Empeltre, Arbequina. Sevillana, Azapa. Cornezuelo, Villa longa.
\end{abstract}

Palabras clave: aceite de oliva, extracción, variedades.

ABSTRACT:

This work determined the feasibility to obtain olive oil from six olive (Olea europuea L) varieties. laken from the Germ Plast Bank of the INPREX-Liniversidad Nacional.Jorge Basadre Grohmann de Tacna. Peri. Characteristics of these varieties were delerminated: Maturity index (an optimun value range of 3-3.7, before the start of maturation stage), Weight in the fresh state (between low and medium), Pulp/seed ratio (Between low and medium). With respect to oil porcentage: Empeltre (24.47\%). Arbequina (15.67\%), Sevillana (24.6\%). Cornezuelo (9.12\%), Villalonga (10.85\%), Azapa $(15.50 \%)$. The extra virgen olive oil obtained from the six varieties studied, a physico chemical analysis was done: acidity index: peroxidation index, value K270): they were found according to the norm. For the physico chemical quantity and quality of produced oil, it has been considered in the following varieties, the oil aptitude in lower order: Empeltre, Arhequina, Sevillana, Azapa, Cornezuelo, Fillalonga.

Keywords: olive oil, extraction, varieties.

Master of Science con Mención en Ciencias y Tecnologia de Alimentos Ingeniero en Indusirias Almentarias. Facultad de Ciencias Agropecuarias. Universidad Nacional Jorge Basadre Grohmani!

= Doctor en Educacion con mención en Gestion Educativa. Magister en Docencia Universitariat. Master of Science con Mencion en Ciencias v Teenologias de Alimentos, Ingeniero en Industrias Alimentarias. Facultad de Ciencras Agropecuarias. Universidad Nacional Jorge Basadre Grohmann 


\section{INTRODUCCIÓN}

El olivo es la especie frutícola más importante del departamento de Tacna, siendo considerado este como el primer productor de este cultivo. En el año 2003 se registró una producción de $23462 \mathrm{~T}$ que equivalen a $61.7 \%$ de la población Nacional y la perspectiva de incrementarse es mayor en tanto debido a que ha se sembrado en año 2002 y 2003 aproximadamente 1000 ha, además cuenta con la productividad más alta del pais que es de 6.4 TM/Ha (Ministerio de Agricultura, 2008). Se tiene también que el $15 \%$ a la producción se destina para la industria del aceite de oliva y el $85 \%$ para procesamiento en aceituna de botija (Díaz, 1988). Contraria a la tendencia mundial donde es mayor la producción de Aceite.

En el INPREX (Instituto de Investigación, Proyección y Extensión Agraria de la UNJBG), posee en el programa de fruticultura, cuyo banco de germoplasma, el cual contiene 31 variedades de olivo edafoclimaticas adaptadas en un área de 0.30 Has.

Una variedad edafoclimáticas adaptada a su medio es en efecto menos susceptible a los factores biológicos y abióticos, lo que tiene una repercusión muy directa en la calidad del aceite de oliva (COI 2002, mencionado por Alférez (2007)).

Por otro lado es necesario de la caracterización (agronómica, fenológica, pomológica y relativa a la calidad del aceite) de las variedades de olivo es conocer mejor los recursos genéticos con vistas a utilizar correctamente las variedades en condiciones edafoclimáticas adaptadas y permitir asi mejorar la calidad de los productos oleicolas (COI 2002, mencionado por Alférez (2007)), del mismo modo que se hace necesario también una caracterización física. química de las variedades del oliva del banco de germoplasma del programa de fruticultura INPREX desde el punto de vista para determinar la idoneidad como materia prima para los diferentes procesos de post cosecha del fruto del olivo, ya que la adaptación edafoclimatica podría generar un cambio de las características de este tipo de la zona de donde es originaria.

Dentro de este marco el objetivo principal es la caracterización tecnológica de las variedades de germoplasma del INPREX y el objetivo específico la deterninación de su Aptitud tecnológica para la elaboración de aceite seis variedades aceiteras del INPREX.

\section{MATERIALES Y MÉTODOS.}

La presente investigación se realizó en los laboratorios de la Facultad de Ingeniería Alimentarias FAIA-UNJBG

\subsection{Materia Prima}

Las muestras de aceitunas se obtuvieron del Banco de Germoplasma del INPREX.
Las variedades de aceitunas que se utilizaron:

-Empeltre

- Liguria

- Sevillana

- Villalong

- Azapa.

\subsection{Materiales y Métodos}

\subsubsection{Materiales}

- Material de vidrio: Vaso de precipitados Erlenmeyer Pipetas Pera de Separación.

- Reactivos: Ioduro de Potasio, Tíosulfato Sódico Ácido acético Solución de almidón Alcohol etílico Éter Etílico. Fenoltaleina.

- Equipos: Soxhlet., Licuadora, Baño Maria, Centrifuga, Frascos de $50 \mathrm{~mL}$.

\subsubsection{Métodos}

- Análisis Materias Primas.

Indice de madurez (Frias 1991)

Peso promedio (Frias 1991)

Relación pulpa/hueso.(Frias 1991)

Contenido de Humedad. (Frias 1991)

Contenido de Aceite (Frias 1991)

- Análisis Aceite de oliva

İndice de Peróxidos. (Frias 1991)

Coeficiente de extinción (Frias 1991)

Acidez Libre. (Frias 1991)

\subsection{Diseño Experimental}

El diseño experimental del presente trabajo se muestra en la Figura 1 y la Figura 2, se muestra el diagrama de operaciones para la obtención del aceite de oliva virgen.

Recolección de materia prima

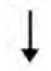

Evaluación de la materia prima

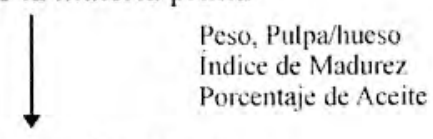

Obtención del aceite de oliva virgen

Evaluación del aceite de oliva virgen

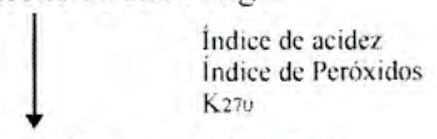

Evaluación Final global de la materia prima

Figura $\mathrm{N}^{\circ}$ I: Diagrama Experimental 


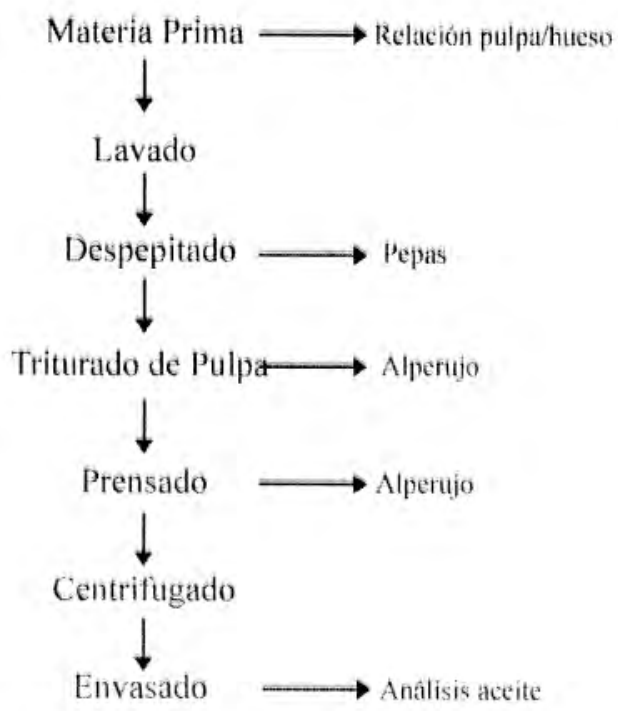

Figura $N^{\circ}$ 2: Diagrama de operaciones para la obtención del aceife de oliva virgen

\section{RESULTADOS Y DISCUSIÓN}

1. Los Resultados de la Evaluación de la materia prima utilizada en la presente investigación se encuentran en la tabla 1.

En la tabla I se puede apreciar que se cuentan con IM de 2.99 y 3.73 que va de piel rojiza a color negra con pulpa blanca, y corresponde a antes de envero; considerado tiempo optimo de recolección. porque la sintesis de aceite alcanza su rendimiento máximo y características sensoriales optimas. (Boskuo 1998).

En cuanto a los pesos de frutos cierta variación y corresponde a la variedad se ubican entre bajo y medio, siendo las de mayo peso las dedicadas a aceituna de mesa, mientras las aceiteras tiene bajo peso y coincide con el propósito de uso que consta en la relación del INPREX.
La relación Pulpa /hueso, muestra la misma tendencia que la del peso anteriormente discutida y cuanto mayor sea será mejor. Siendo las mayores en variedades utilizadas para mesa.

La relación pulpa/hueso se encuentra entre media y baja.

En cuanto a la cantidad de aceite cabe señalar corresponde a la variedad aceitera empeltre y es también señalado en España como la mejor variedad para aceite.

Las variedades Sevillana a pesar tener un alta relación pulpa hueso y tiene una alta cantidad de aceite y tiene un doble función como materia prima para aceite y aceituna de niesa.

2. Característicos del aceite de oliva oblenido de las mulestras.

Estos resultados se encuentran en la Tabla 2, donde se observa que todos los valores del los aceites de oliva se encuentran en los parámetros de calidad.

\section{CONCLUSIONES}

4.1. Por la cantidad y la calidad físico química de aceite producido se ha considerado las siguientes variedades con aptitud aceiteras en orden decreciente: Empeltre, Arbequina, Sevillana, Azapa, Cornezuelo. Villa longa

\section{RECOMENDACIONES}

1. Realizar pruebas sensoriales para una evaluación integral

2. Realizar las pruebas en diferentes estados de madurez de las diferentes variedades.

3. Realizar la evaluación de otras variedades de aceitunas del Banco de Germoplasma del INPREXUNJBG.

Tabla 1. Caracteristicas del Fruto

\begin{tabular}{|c|c|c|c|c|c|c|}
\hline & Empeltre & Arbequina & Sevillana & Cornezuelo & Villalonga & Azapa \\
\hline $\begin{array}{l}\text { Índice de } \\
\text { Madurez }\end{array}$ & 2.99 & 3.73 & 3.28 & 3.25 & 3.32 & 3.16 \\
\hline $\begin{array}{l}\text { Peso } \\
\text { Promedio(gr.) }\end{array}$ & 4.074 & 2.097 & 7.414 & 7.575 & 5.06557 & 6.983 \\
\hline $\begin{array}{l}\text { Relación } \\
\text { Pulpa-hueso }\end{array}$ & 4.9940 & 3.964 & 5.492 & 6.468 & 5.160 & 3.6910 \\
\hline $\begin{array}{l}\text { Contenido } \\
\text { Aceite (\%) }\end{array}$ & 24.47 & 15.67 & 24.61 & 9.72 & 10.85 & 15.50 \\
\hline Agua (\%) & 62.11411 & 60.27 & 64.38 & 53.15 & 52.48 & 57.07 \\
\hline
\end{tabular}

Tabla 2. Características físico-química del aceite de oliva

\begin{tabular}{lllllll}
\hline Análisis & Empeltre & Liguria & Sevillana & Villalonga & Cornezuelo & Azapa \\
\hline Acidez Libre (\%) & 0.141 & 0.1962 & 0.6720 & 0.1675 & 0.4471 & 0.1691 \\
Índice Peroxido & 2.6062 & 2.6 & 3.35 & 1.25 & 3.56 & 1 \\
Coeficiente K $_{270}$ & 0.1779 & 0.1423 & 0.1496 & 0.1103 & 0.08245 & 0.1867 \\
\hline
\end{tabular}




\section{REFERENCIAS BIBLIOGRÁFICAS}

Alférez, R; Robles, M. (2007). Caracterización morfológica de trece variedades de Olivo (Olea europeae L) introducidas en el germoplasma del INPREX-Tacna. COIN-UNJBG

Aranda, Z R. (2001) .Ingeniería en decantadores para un estudio de factibilidad de una planta procesadora de aceite de oliva en la irrigación de la Yarada. UNJBGFAIA. Tesis para optar el Titulo Profesional de ingeniera en industrias Alimentarias, UNJBG. Tacna.

Boskou Dimitrios (1998). Química y Tecnología de aceite de Oliva. Editorial Mundi prensa .Madrid. España

Díaz, A (1988) Diagnóstico de la producción de aceitunas de mesa en el Perú.

De Florio, E. (2009). Visita de Campo al Banco de Germoplasma del IMPREX. Informe no publicado.

Earlie R, L. (1998) Ingeniería de los Alimentos. Editorial Acriba. Zaragoza.

Frías, L.; García-Ortiz, A.; Hermoso, M.; Jimenez, A.; Llavero, M.P.; Morales, J.; Ruano, T.; Uceda, M. (1991). Analista de Laboratorio de Alzamara. Consejería de Agricultura y Pesca de Andalucía. .Colección Apuntes $N^{\circ} 6 / 1991$. Sevilla.

Lanchipa Bergamini Liliana (2003) Evaluación de la Calidad Químico -Sensorial de los aceites de Oliva virgen mono varietales de Tacna. Tesis Optar grado de Magíster en Ciencia y Tecnología de Alimentos. ESPG-

\section{UNJBG. Tacna. Perú.}

Leo, R, E. (2000) Diagnóstico de la Calidad del Aceite de Oliva Tacneño Virgen según el Proceso Productivo. Tesis Optar Titulo de Ingeniero. FAIA-UNJBG.

Loussert, R; Brousse, G. (1980). El Olivo. Madrid.

Conservas Kilimanjaro (2009) Revista virtual. Disponible en

www.conservaskilimanjaro.com/aceitunas.php

Enciclopedia Libre Universal. Olivo. http://enciclopedia.us.es/index.php/Olivo. 24-04-09. Revisado el día 24 de Abril del 2009 de la página

FAO. www.fao.org Página Web de la FAO

International olive oil

http://www.internationaloliveoil.org/resgen/esp/Espa $\%$ $\mathrm{C} 3 \% \mathrm{~B} 1 \mathrm{a} /$ pomologica.pdf

Ministerio de Agricultura Boletín Mensual 2009.

\section{Correspondencia:}

Enrique Alfonso De Florio Ramirez

Ciudad Universitaria - Fundo "Los Granados"

Av. Miraflores s/n. Tacna. Perú

enriquef11@hotmail.com

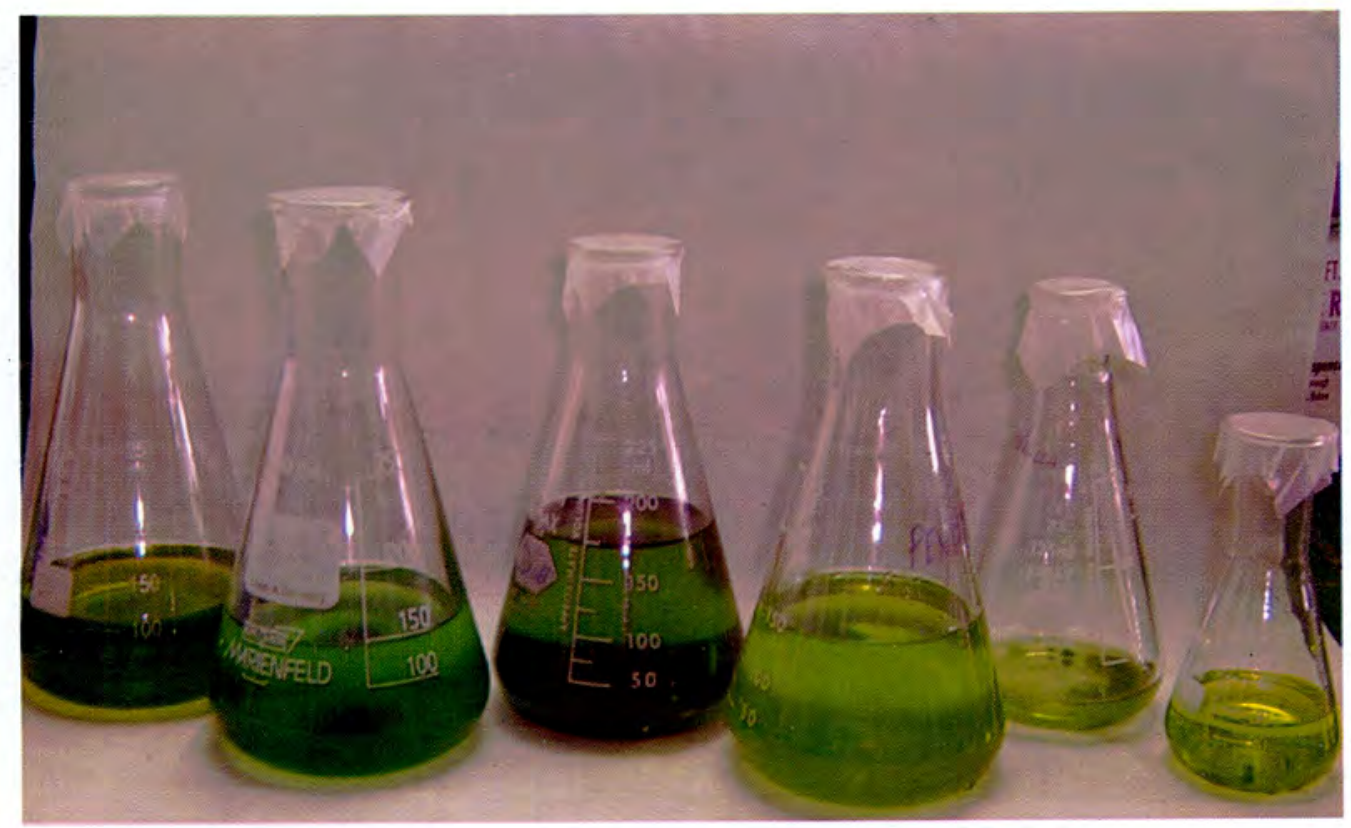

Aceite de Oliva de Seis Variedades de Aceituna Negra del Banco de Germoplasma del Instituto de Investigación, Proyección y Extensión Agraria de la Universidad Nacional Jorge Basadre Grohmann de Tacna 